\title{
A new method to predict meteor showers
}

\section{Application to the Leonids}

\author{
J. Vaubaillon ${ }^{1}$, F. Colas ${ }^{1}$, and L. Jorda ${ }^{2}$ \\ 1 Institut de Mécanique Céleste et de Calcul des Éphémérides, Observatoire de Paris, 77 avenue Denfert-Rochereau, \\ 75014 Paris, France \\ e-mail: vaubaill@imcce.fr \\ 2 Laboratoire d'Astrophysique de Marseille, Site Pereisc, 13376 Marseille Cedex 12, France
}

Received 30 December 2004 / Accepted 17 March 2005

\begin{abstract}
Our model of meteor shower forecasting (described in Paper I) is applied to the Leonid shower. This model is based on the "dirty snowball" model of comets, and on heavy numerical simulation of the generation and evolution of meteoroid streams. The amount of dust emitted by comet 55P/Tempel-Tuttle is computed. A statistical weight is associated to each simulated particle. This weight represents the real amount of meteoroids released by the comet. Particles close to the Earth are examined. There is no unique set of initial conditions (velocity and angle of ejection) for them to reach the Earth at the time of the shower. The shape of the metoroid stream projected on the ecliptic is not elliptical, due to non-gravitational forces and ejection processes. The mixing of particles is revealed to be very efficient. A comparison between observations and predictions of Leonid meteor showers is done. The time of maximum is found to be very accurate, except for certain years (1999 in particular). This problem is common to all models. The level of the predicted shower is obtained through a fit of the size distribution index $s=2.4 \pm 0.1$. This model provides a unique opportunity to derive cometary parameters from meteor shower observations. Leonid meteor shower forecasts are provided for years up to 2100. The next storm is expected in 2034.
\end{abstract}

Key words. meteors, meteoroids - comets: general - ephemerides

\section{Introduction}

A meteor storm is a very rare event, and is a unique opportunity to study several aspects of meteors (altitude, light curve, spectroscopy, link with comets, orbit etc. See e.g. Jenniskens 2002b). Such a phenomenon occurs when the Earth collides with a meteoroid stream (Kresak 1993). The difficulty of forecasting of meteor storm comes, among other parameters, from the difficulty of observing meteoroid stream. Sykes et al. (1986) were the first to directly detect a meteoroid stream.

Several methods were developed to predict Leonid meteor storms. The evolution of the meteor stream ejected by the parent body comet 55P/Tempel-Tuttle was done by Williams (1997), and the Leonid meteor storm were first correctly predicted by McNaught \& Asher (1999). The number of meteors impacting the Earth has also been studied by Lyytinen \& Van Flandern (2000) and Lyytinen et al. (2001). Complete observations were done by the Leonid MAC Campaigns (Murray et al. 1999; Gural \& Jenniskens 2000; Jenninskens 2001, 2002a) and IMO (Arlt \& Brown 1999; Arlt et al. 1999; Arlt \& Gyssens 2000; Arlt et al. 2001, 2002). However, none of these models take into account the photometry information of the parent body.
This paper is the application of a new method of meteor shower forecasting (described in Vaubaillon et al., Paper I). This method is based on a numerical simulation of the formation and evolution of meteoroid streams, coupled with information deduced from photometric observations of the parent body. We performed a link between real and simulated meteoroids, in order to compute a real particle density in the neighbourhood of the Earth at the time of the maximum of a shower. Paper I described the general method to compute the real number of particles ejected by the comet and the statistical weight set to each simulated meteoroid. This paper is the application of this method to the Leonid meteor showers. Preliminary results were shown (Vaubaillon \& Colas 2002; Vaubaillon 2002; Vaubaillon et al. 2003, 2004), but here we publish the complete results of our approach and Leonid forecastings for the coming 100 years.

In Sect. 1 we present how the simulation is run and the application of our model to comet 55P/Tempel-Tuttle. Then we provide a statistical analysis of the results (Sect. 2) and a comparison between predictions and observations (Sect. 3). A discussion follows in Sect. 4. 
Table 1. Values of variables deduced from observations of comet 55P/Tempel-Tuttle. $r_{\mathrm{n}}$ is taken from Hainaut et al. (1998), $m_{\mathrm{H}}$ from Beech et al. (2001) and $[A f \rho]$ from Lamy et al. (1998). $Q_{\mathrm{H}_{2} \mathrm{O}}(q)$ is deduced from $m_{\mathrm{H}}$ thanks to Jorda et al. (1992)'s formula, and $f$ from Crifo \& Rodionov (1997), Appendix A, Eq. (9).

\begin{tabular}{ccc}
\hline \hline Symbol & Name & Value \\
\hline$r_{\mathrm{n}}$ & radius of nucleus & $1.8 \mathrm{~km}$ \\
$m_{\mathrm{H}}$ & absolute magnitude & 8.5 \\
$q$ & perihelion distance & $0.997 \mathrm{AU}$ \\
{$[A f \rho](q)$} & {$[A f \rho]$ at perihelion } & $78.9 \mathrm{~cm}$ \\
$Q_{\mathrm{H}_{2} \mathrm{O}}(q)$ & gas production rate & $3.715 \times 10^{28} \mathrm{~mol} \mathrm{~s}^{-1}$ \\
$f(q)$ & fraction of active area & 0.24 \\
\hline
\end{tabular}

\section{Simulating a meteoroid stream}

\subsection{Model and hypotheses}

Our goal is to simulate the formation and evolution of a meteoroid stream in order to derive ephemerides of meteor showers. The physical model supposed to represent the processes has to be as complete as possible. It has to take into account the initial conditions and the dynamics of a meteoroid in the Solar System.

The complete list of hypotheses considered here was provided in Paper I. However, we recall here those relevant for this paper. We will hereafter consider that:

1. meteoroids are ejected by a cometary parent body;

2. the comet emits meteoroids while its heliocentric distance is less than 3 ua;

3. the ejection is done in the sunlit hemisphere;

4. the ejection velocity is given by Crifo \& Rodionov (1997)'s model;

5. the dust particles are spherical, homogeneous, of density $\rho_{\mathrm{g}}=2000 \mathrm{~kg} \mathrm{~m}^{-3}$

6. the particles giving meteors have radii greater than $0.1 \mathrm{~mm}$ (Hughes 1995);

7. their size distribution follows a power law, of index $s$;

8. their orbits are controled by the gravity of the Sun and planets of the solar system, and by non-gravitational forces such as radiation pressure, Poynting-Robertson drag and Yarkovky's effect ${ }^{1}$.

\subsection{Features of the parent body}

To use the photometric information of the comet, we reproduce in Table 1 some parameters measured on 55P/Tempel-Tuttle.

Table 2 summarizes the values of all useful parameters deduced from our calculations (see Paper I), when applied to comet 55P/Tempel-Tuttle.

The $f(q)$ parameter differs by an order of magnitude compared to the one provided by Beech et al. (2001), but is reasonable and our method is more complete. We stress the importance of the $s$ factor in these data (see Paper I). Indeed, a change of $s$ will cause a change in all dust production parameters. We will discuss the relevance of this parameter in Sect. 3 .

\footnotetext{
1 This effect was taken into account only for particles larger than $0.1 \mathrm{~m}$.
}

Table 2. Parameters deduced from calculations detailed in Paper I, when applied to comet 55P/Tempel-Tuttle.

\begin{tabular}{ccc}
\hline \hline Symbol & Name & Value \\
\hline$Q_{\mathrm{H}_{2} \mathrm{O}}(q)$ & gas production rate & $3.715 \times 10^{28} \mathrm{~mol} \mathrm{~s}^{-1}$ \\
$Q_{m_{\mathrm{H}_{2} \mathrm{O}}}^{t}$ ot & Total gas mass loss & $2.64 \times 10^{10} \mathrm{~kg}$ \\
$A_{\mathrm{B}}$ & Bond albedo & 0.3 \\
$A(\phi)$ & albedo & 0.24 \\
{$\left[a_{1} ; a_{2}\right]$} & All particles radius range & {$\left[10^{-6}, 10^{-1}\right] \mathrm{m}$} \\
{$\left[a_{1}^{\prime} ; a_{2}^{\prime}\right]$} & meteor radius & {$\left[10^{-4}, 10^{-1}\right] \mathrm{m}$} \\
$s$ & size population index & 3.0 \\
$N$ & Normalizing factor & $2 \times 10^{-12}$ \\
$K$ & coefficient & $4.42 \times 10^{-17}$ \\
$f^{\mathrm{t}}$ & time of outgasing & $\simeq 3.60 \times 10^{7} \mathrm{~s}$ \\
$f^{r_{\mathrm{h}}}$ & heliocentric distance factor & 0.658 \\
$z_{\mathrm{g}}\left(a_{1}, 0, q\right)$ & local dust production rate & $3.23 \times 10^{11} \mathrm{~s}^{-1}$ \\
$z_{\mathrm{g}}\left(a_{1}, 0,3\right)$ & idem & $3.23 \times 10^{-4} \mathrm{~s}^{-1}$ \\
$z_{\mathrm{g}}\left(a_{1}^{\prime}, 0, q\right)$ & idem & $3.23 \times 10^{5} \mathrm{~s}^{-1}$ \\
$z_{\mathrm{g}}\left(a_{1}^{\prime}, 0,3\right)$ & idem & $3.23 \times 10^{4} \mathrm{~s}^{-1}$ \\
$z_{\mathrm{g}}\left(a_{2}^{\prime}, 0, q\right)$ & idem & $3.42 \times 10^{10} \mathrm{~s}^{-1}$ \\
$z_{\mathrm{g}}\left(a_{2}^{\prime}, 0,3\right)$ & idem & $3.42 \times 10^{-5} \mathrm{~s}^{-1}$ \\
$Q_{\mathrm{g}}\left(q, a_{1}^{\prime}, a_{2}^{\prime}\right)$ & total dust production rate & $1.64 \times 10^{8} \mathrm{~s}^{-1}$ \\
$N_{\mathrm{g}}^{\mathrm{tot}}\left(a_{1}^{\prime}, a_{2}^{\prime}\right)$ & total dust production & $3.90 \times 10^{15}$ \\
$z_{\mathrm{m}}\left(q, a_{1}^{\prime}, a_{2}^{\prime}\right)$ & dust mass loss rate & $2749 \mathrm{~kg} \mathrm{~s}^{-1}$ \\
$M_{\mathrm{g}}\left(a_{1}^{\prime}, a_{2}^{\prime}\right)$ & total dust mass loss & $6.52 \times 10^{10} \mathrm{~kg}$ \\
$M_{\mathrm{g}}\left(a_{1}, a_{2}\right)$ & idem, for $\left[a_{1}, a_{2}\right]$ & $6.53 \times 10^{10} \mathrm{~kg}$ \\
$R$ & dust to gas mass ratio & 2.47 \\
\hline$R$ & & \\
\hline
\end{tabular}

Sometimes the cumulative mass distribution index ( $b$ or $s_{\mathrm{mc}}$ ) is measured on comae (Fulle et al. 2000). There is also a population index for meteors $(r)$. We refer the reader to Paper I, Appendix C for the relationship between these different indices and the numerical applications. Extreme values of $r$ were reached in 1998 and 2002 (Brown \& Arlt 1998; Arlt et al. 2002). Numerical values provided in Table 2 assume that each index is constant during a single meteor shower. Some variations may occur, as discovered by Jenninskens (2001).

We find in Table 2 some values already found by different approaches. Jenninskens (2001) computed:

- total dust mass ejected by 55P/Tempel-Tuttle: $2.6 \pm 0.7 \times$ $10^{10} \mathrm{~kg}$;

- total gas mass ejected by comète $55 \mathrm{P} /$ Tempel-Tuttle: $1.1 \pm$ $0.7 \times 10^{10} \mathrm{~kg}$;

- dust to gas mass ratio: $2.4 \pm 1.7$.

In a later work (Jenniskens 2002a) these values were revised, but the order of magnitude remains identical.

The major of mass ejected is carried by large particles, and is the reason why tiny particles play a small role in computing $R$. A decreasing size index will increase this ratio: it goes to 3.6 if $s=2.2$.

As partices are ejected on a cometary arc of the orbit (assumptions 1-3), the position - velocity of the parent body has 
to be computed. This ephemeris is done by considering the gravitational influence of all the planets of the solar system, as well as non-gravitational forces. The non-gravitational parameters (Marsden 1969) for each perihelion return were provided by P. Rocher (IMCCE, personal communication).

The JPL planetary model DE406 was used and the cometary ephemeris is saved each Julian day of the outgasing process. In total, 29 perihelion returns of comet 55P were taken into account. The dates of perihelion passages are from 1300 to 1998, from 604 to 802 AD, and 1001. The choice of these returns is motivated by the fact that meteor storms are caused by young trails (McNaught \& Asher 1999), and we also wished to examine the behaviour of trails ejected more than a millenium ago. The 1001 return is an intermediate between these 2 cases.

\subsection{Integration of particles}

The initial conditions of meteoroids are given by the cometary ephemerides and the ejection velocity, provided by the Crifo \& Rodionov (1997) model (see Paper I for further details). Planetary ephemerides are also given by the DE406 model, and non-gravitational forces are taken into account (assumption 8, see Paper I, Fig. 2 for further details). The algorithm used here is a 15th order Radau (Everhart 1985).

There are more small particles than large one (assumption 7). The amount of dust emitted by a comet during one perihelion return is so large that is it impossible to simulate. In order to sample the size range of meteoroids giving rise to meteors, 5 bins were defined: $[0.1 ; 0.5],[0.5 ; 1],[1 ; 5],[5 ; 10]$ and $[10 ; 100] \mathrm{mm}$ (see also Paper I). $N_{\mathrm{p}}=5 \times 10^{4}$ particles were simulated in each bin, for a total of $\simeq 7 \times 10^{6}$ simulated particles. The simulations were run on 10 to 50 parallelized processors at CINES (Montpellier, France). This parallelized work was motivated by the fact that a single numerical integration (one perihelion return and one size bin) lasts several hours to several days on a mono-processor computer. In all, the simulation would have taken almost a year, but was reduced to a week by the parallelisation.

Following the work of Göckel \& Jehn (2000) we define a first space criterion (see Paper I) to select the nodes of meteoroids that are close to the Earth, hereafter called Near Earth Meteoroids (NEM). This space criterion corresponds to a time criterion $\Delta T \simeq 0.002 \mathrm{yr}$, at Earth-Leonid relative velocity. This method allows us to perform meteor storm predictions without any previous knowledge of the date of annual showers. As seen in Paper 1, these NEM do not necessary collide with the Earth, and we were forced to use a second space criterion $\delta x$ to select those meteoroids considered as impacting the planet. The second space criterion corresponds to a time criterion $\delta t=1 \mathrm{~h}$, at Earth orbital speed.

We first examine the features of particles selected by the first criterion.

\section{Analysis of impacting particles}

In this section we analyse the results of the first space criterion, and discuss the features of NEM. We will focus here on the 1866 trail, as it is representative of a "young" trail, and shows many interesting features. Preliminary results have already been presented (Vaubaillon \& Colas 2002; Vaubaillon 2003), but we show here all the results in detail.

\subsection{Feature of ejection velocity}

Figure 1 shows the nodes of 3 size bins of NEM, ejected during the 1866 return of the comet, in 1998, 1999 and 2001. The images are similar to Wu \& Williams (1993). The ejection velocity is shown as a function of time of ejection.

From this figure, we can see that:

- There is an efficient mix of particles in the encountered shower, due to the ejection velocity and non-gravitational effects.

- The shape of the projection of the trail on the ecliptic is not elliptical, as shown by Asher (2000). It is similar to data of McNaught \& Asher (1999): from the center, the spatial density of meteoroids decreases faster in the solar direction than in the opposite direction, because of nongravitational forces (especially the radiation pressure). This is why McNaught \& Asher (1999) defined a ZHR model as a function of the sign of $r_{\mathrm{E}}-r_{\mathrm{D}}$. We want to avoid such a dependance in the calculation of the ZHR value; this is the role of the second space criterion (see Paper I).

- The width of the stream does not vary much from one year to another.

- Large particles are concentrated in the "head" of the stream, as a consequence of low ejection velocity and low efficiency of radiation pressure.

- The required ejection velocity increases with time, rising from $7 \mathrm{~m} \mathrm{~s}^{-1}$ to $15 \mathrm{~m} \mathrm{~s}^{-1}$. This is normal since low velocity particles stay in the vicinity of the nucleus.

- The scatter of the velocity value decreases when mass increases. This effect was not expected and shows that there is not a unique solution.

- Particles are preferably ejected at high heliocentric distances, where the ejection velocity is lower than at perihelion. But this effect disappears with time. For particles close to the comet (years 1998-1999), there is clearly a gap in low velocities at perihelion. This is understandable in the Keplerian case: a high ejection velocity is required to dramaticaly change the node of a particle. This is especially true for the Leonids because the perihelion and descending nodes are very close.

- A high ejection velocity is required to move large particles unto orbits different from that of the parent body. Thus large meteoroids selected a few years after the comet return were emitted close to perihelion.

\subsection{Feature of angles of ejection}

Angles of ejection from a subsolar point at the time of ejection, in $(a)$ and perpendicular to $(b)$ the cometary orbital plane are chosen to equally cover the sunlit hemisphere (assumption 3 ). 

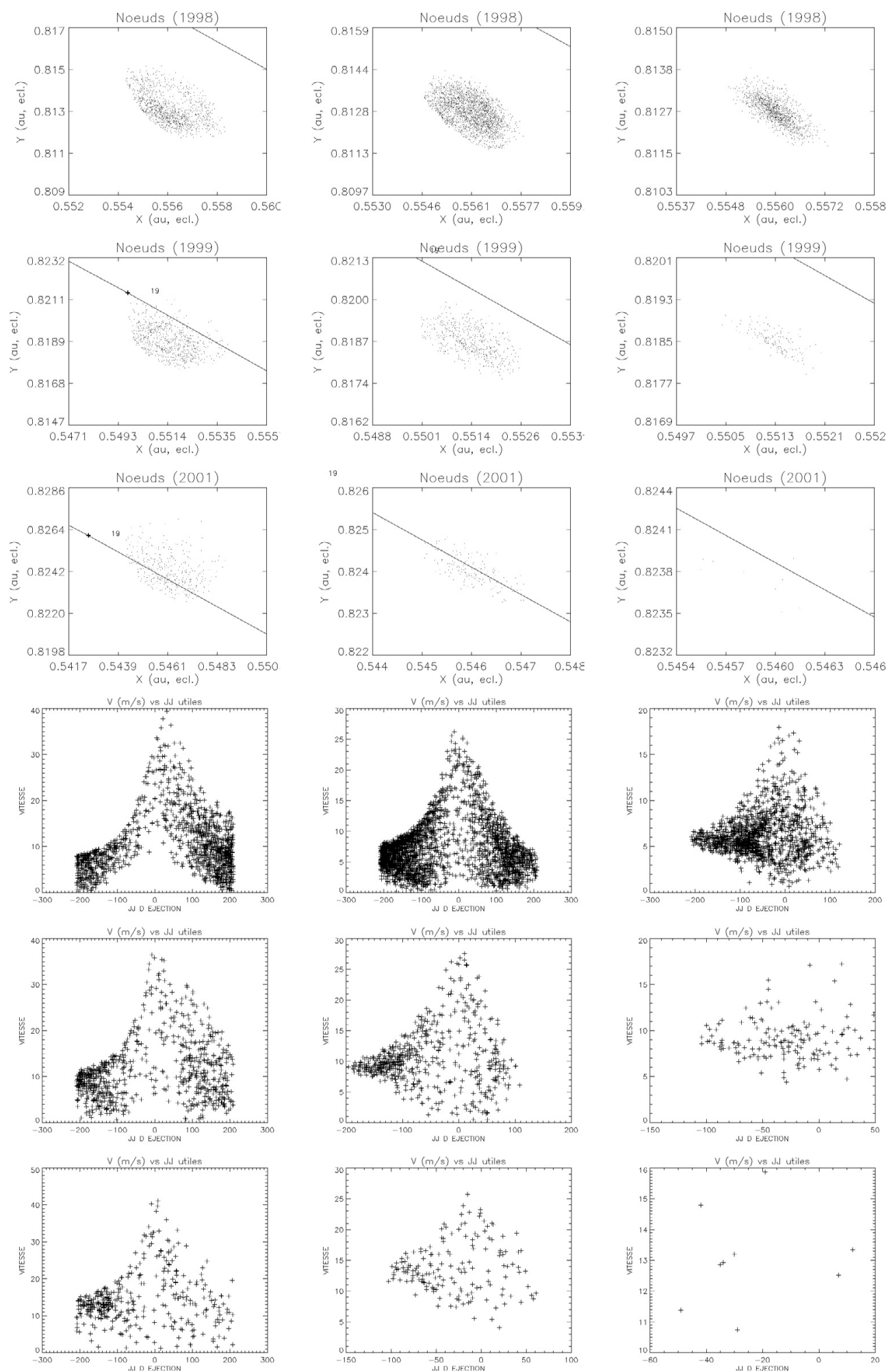

Fig. 1. Nodes and histograms of ejection velocity of NEM ejected during the 1866 perihelion return. Left: [0.1-0.5] mm size bin, center: [0.5-1] mm size bin, right: [1-5] mm size bin. In the images of the nodes, the line represents the trajectory of the Earth. Frame is heliocentric, ecliptic J2000. In the diagrams showing the ejection velocity as a function of day of ejection, 0 is perihelion.

Figure 2 shows the repartition of angles of ejection for the same particles and years as Fig. 1.

For both angles we observe the relaxing of constraints with time. The distributions tend to flatten, reflecting the mixing of particles in the section of the streamfar from the comet.
In the orbital plane, we can observe that:

- There is clearly a gap of negative angles for small particles around perihelion. This is understandable as small particles are very sensitive to the ejection process, and the cometary node is inside the Earth's orbit. Such particles cannot reach 

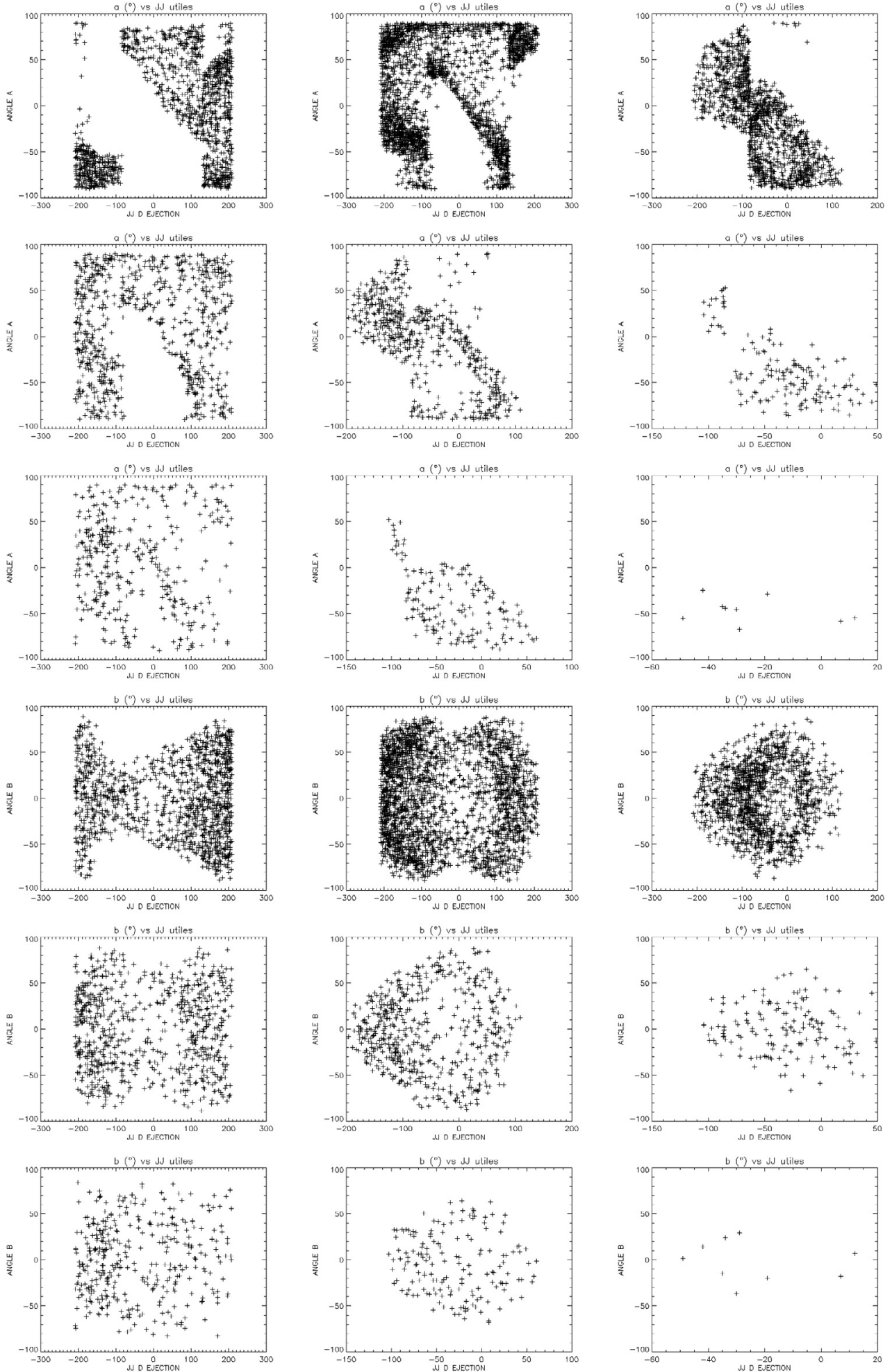

Fig. 2. Feature of angle of ejection for the same particles as Fig. 1, versus time of ejection ( 0 is perihelion). First 3 rows are the distribution of the angle of ejection in the orbital plane of the comet, in 1998, 1999 and 2001. Last 3 rows are angle of ejection in the direction perpendicular to the orbital plane for the same years. Left: [0.1-0.5] $\mathrm{mm}$ size bin, center: [0.5-1] mm size bin, right: [1-5] mm size bin. See Sect. 2.2 for further details.

the Earth if ejected in the "wrong" direction. Note that the node itself can change, according to the direction of ejection.
- Large particles that are less sensitive to ejection (lower relative speed) however suffer from the selection process. The gaps are not the same as small ones. This again comes from 

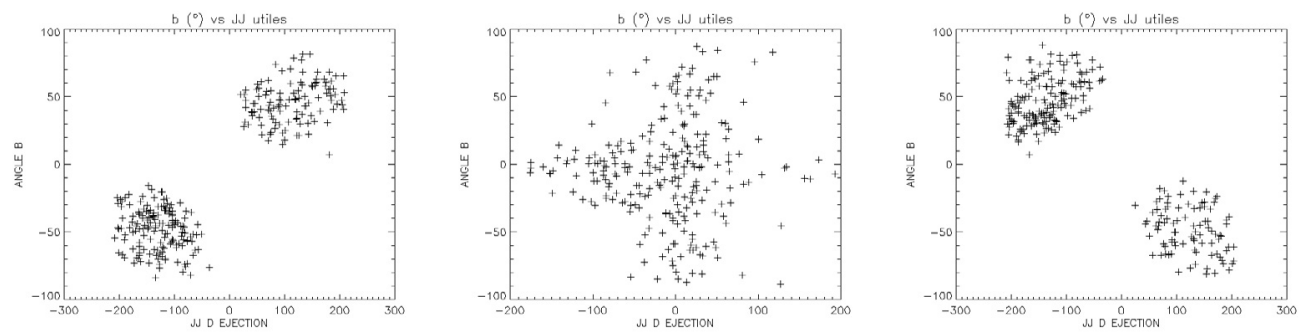

Fig. 3. Angles of ejection of particles successively encountered by the Earth during a meteor shower $([0.1 ; 5] \mathrm{mm})$.

the mixing of different populations, due to differences in orbital period.

- If the 1866 stream well represents the other ones, the particularities underlined here always appear, but at different times. This reflects the stream composition along the cometary orbit.

- The rotation of the cometary nucleus is fast compared to its orbital period. Thus any equatorial jet effect is quickly averaged and then becomes impossible to recognize here.

In the direction perpendicular to cometary orbital plane:

- There is no selection as to which side particles are ejected.

- There are fewer constraints than for the angle in the orbital plane.

- When contraints exist, ejections in the plane are prefered. High values of $b$ are absent for small particles ejected before perihelion.

- Large particles are preferably ejected in the plane for the years the farer from the perihelion return. But this also reflects the fact that a high ejection velocity is required.

- Here again there can be a time decay for other streams resulting in such features.

- The three images of Fig. 3 show the angles of ejection of selected particles that the Earth encounters successively. This corresponds, on the images of Fig. 1 representing the nodes of meteoroids, to particles located right, center and left of the cloud. We see a strong selection here, because of the geometry of the ejection. The central part of the stream is filled with particles preferentially ejected in the plane. This is not true for those ejected near perihelion.

These results show that during a meteor shower, the Earth samples different kinds of particles, from directions of ejection.

We now compute the date of maximum of the shower and $Z H R$ values, and compare them with observations.

\section{Comparison between predictions and observations}

For this comparison we uses two sources of observations: the results provided by IMO (Arlt \& Brown 1999; Arlt et al. 1999; Arlt \& Gyssens 2000; Arlt et al. 2001, 2002) and those by the Leonid MAC missions (Jenniskens 2002a).

\subsection{Time of maximum}

As we saw in Paper I, the time of maximum is computed as the median position of the nodes. Table 3 provides the comparison between calculations and observations done by IMO.

We can see a good agreement, with a difference of several to a few tens of minutes.

The greatest differences are found for years 1999 and 2000, and are also found in other works. This led us to ask if another still-unknown stream had been encountered (Vaubaillon \& Colas 2002), but without a definite answer.

The year 2000 is particular in the way that the maximum difference is recorded. Note that the 1333 stream encounter is only mentioned in this work. Observers report a plateau in the activity, which lasted about one hour, centered on the time mentioned in Table 3. That is why the time of maximum is not well constrained. Note that two other streams should be observable: the one of 1333 and 1767 . However, adding the 1333 stream tends to make the maximum later, instead of earlier. These two streams were also supposed to have a small contribution. As a consequence the different contributions of these two streams to the shower were hard to detect.

It is interesting to stress again the differences between models. Lyytinen et al. (2001) did not consider any ejection velocity process, but took into account the Yarkovsky's force for all particles. As their and our results agree about the time of maximum we can suppose that the true case lies between the two.

\subsection{ZHR computation}

All meteor forecasting methods are based on observations used to fit a model. In theory, our apporach does not need such a fit (reflecting the strength of this approach). However, since all cometary parameters are not totally known, we also have to take into account the past showers in our predictions. But the only parameter fitted here is $s$, by minimising the $\chi^{2}$. As this parameter is the most critical, there is no need to fit other parameters. In this way we obtain some information about the population index of the cometary dust by observing meteor showers. This makes this work unique compared to other methods. Figure 4 shows our results.

In the ideal case, all the points are situated on the line (equality between computation and observations). The points below the line under-estimate the level, and inversely for the points above.

The fit is correct for recent observations (that is, since 1998), with differences of a few hundreds of meteors for $Z H R$. 
Table 3. Comparison between computed and observed time of maximum. Time is given in UT. Observations are from IMO. Comparisons are with other models from McNaught \& Asher (1999); Lyytinen (1999); Lyytinen \& Van Flandern (2000); Lyytinen et al. (2001). See text for further details about the 1333 trail encountered in 2000.

\begin{tabular}{cccccccccccccc}
\hline \hline Year & Stream & \multicolumn{3}{c}{ Observations } & \multicolumn{1}{c}{ McN. \& A. } & \multicolumn{1}{c}{ L. \& V. F. } & \multicolumn{3}{c}{ This work } \\
& & Day & Hr & Min & Day & Hr & Min & Day & Hr & Min & Day & Hr & Min \\
\hline 1998 & 1333 & 17 & 1 & 55 & - & - & - & - & - & - & 17 & 1 & 29 \\
1999 & 1899 & 18 & 2 & 2 & 18 & 2 & 08 & 18 & 2 & 10 & 18 & 2 & 13 \\
1999 & 1932 & 18 & 1 & 43 & 18 & 1 & 44 & 18 & 1 & 40 & 18 & 1 & 49 \\
2000 & 1333 & $(?)$ & $(?)$ & $(?)$ & - & - & - & - & - & - & 18 & 4 & 59 \\
2000 & 1733 & 18 & 3 & 16 & 18 & 3 & 44 & 18 & 3 & 40 & 18 & 3 & 48 \\
2000 & 1866 & 18 & 7 & 12 & 18 & 7 & 51 & 18 & 7 & 50 & 18 & 7 & 57 \\
2000 & 1932 & 17 & 8 & 7 & 17 & 7 & 53 & 17 & 8 & 00 & 17 & 7 & 55 \\
2001 & 1767 & 18 & 10 & 33 & 18 & 10 & 01 & 18 & 10 & 28 & 18 & 10 & 6 \\
2001 & 1866 & 18 & 18 & 16 & 18 & 18 & 19 & 18 & 18 & 26 & 18 & 18 & 25 \\
2001 & 1699 & 18 & 18 & 2 & - & - & - & 18 & 18 & 03 & 18 & 17 & 59 \\
2002 & 1767 & 19 & 4 & 10 & - & - & - & 19 & 04 & 02 & 19 & 4 & 02 \\
2002 & 1866 & 19 & 10 & 47 & 19 & 10 & 36 & 19 & 10 & 44 & 19 & 10 & 48 \\
\hline
\end{tabular}
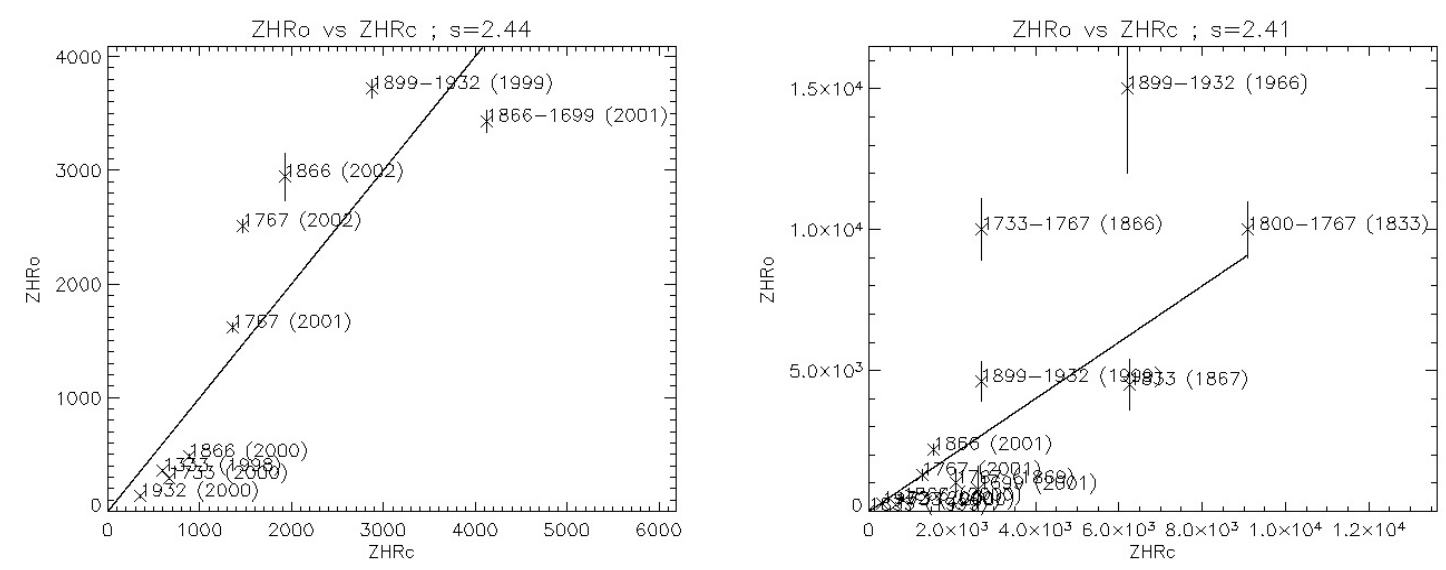

Fig. 4. Comparison between observed $Z H R_{\mathrm{o}}$ and computed $Z H R_{\mathrm{c}}$, by fitting $s$ parameter, from IMO observations (left) since 1998, and by results given by Jenniskens (2002a). The numbers refer to the stream, and the one in brackets the year of observation. The equation of the line is $Z H R_{\mathrm{o}}=Z H R_{\mathrm{c}} . s$ values are deduced by a $\chi^{2}$ test.

However, we often took into account the contribution of several streams at the same time. It happened that several clouds overlap, as seen in the Pi-Puppids case (Vaubaillon \& Colas 2005). It is then hard to separate each component (Uchiyama 2002). In particular we mention the non-negligeable contribution of the 1932 stream to the 1999 shower, which was not noticed before.

For historical observations, the agreement is good for the year 1833, but not for 1866 and 1966. However the fit has not been done from observations themselves $(Z H R=50000$, Brown 1999), but from values deduced by Jenniskens (2002a). This choice comes from the uncertainties in the reported values. Such high ZHR led McNaught \& Asher (1999) to overestimate the Leonid storms.

We have also found that some very old streams have contributed to the 1833 shower. Table 4 gives each contribution.

It is encouraging to find a $s$ value compatible with cometary observations. Fulle et al. (2000) measured $s=2.6$, and literature gives similar values, often between 2.5 and 3.5 , but these values are computed for smaller dust size, i.e. corresponding to those scattering the visible wavelength and observed in cometary dust tails. Campbell-Brown \& Koschny (2004) working on the ablation process of meteoroids developed a fragmentation model. In this model particles are composed of grains maintained together by a "glue". They tried a power law distribution of the grains, and found values up to 2.4. However this distribution does not represent exactly the same quantity as here, since it deals with the internal structure of the meteoroids.

Our fit giving $s=2.4 \pm 0.1$ is satisfactory. However the meteor population index is not always equal to $r=1.6$ (see Paper I, Appendix C). We recall that ejection processes and non-gravitational forces efficiently mix the particles. The same stream giving rise to several storms does not always show the same $r$ value. The best example is the 1866 stream, which revealed a high value in $2002(r=2.9)$, whereas this was not the case in 2000 ( $r=2.05$ ) (Arlt \& Gyssens 2000; Arlt et al. 2002).

\subsection{The 1998 shower}

The 1998 shower is famous for many reasons. First because of the 16 hour difference between predictions and observations (Asher et al. 1999). Second, the explanation of the phenomenon has several versions. Asher et al. (1999) determined that the 1333 stream only was responsible for the shower. Later, Brown \& Arlt (2000) proposed that it was caused by several stream 
Table 4. Contributions of very old streams to the 1833 shower, that were not noticed yet.

\begin{tabular}{ccccc}
\hline \hline Year & stream & D/M & h:mn & ZHR \\
\hline 1833 & 1333 & $13 / 11$ & $11: 26$ & 70 \\
1833 & 1666 & $13 / 11$ & $15: 50$ & 30 \\
1833 & 1699 & $13 / 11$ & $15: 53$ & 30 \\
\hline
\end{tabular}

from the XIVth centuary. Lyytinen (1999) saw the influence of the 801 stream.

We have examined the problem, performing the simulations of old streams: 602 to 802 . The cometary orbit somewhat differs from that taken by Lyytinen (1999), because the perihelion return was not exactly the same. This is due to some differences in the computation of the cometary orbit on relatively long time scales (a millenium). We see here a limit to many (if not all) models: the cometary orbit does not allow one to perform accurate predictions after a long time, unless there are some reported observations (see also Sect. 4).

Our results suggest a major contribution of the 1300 and 1333 streams ( $Z H R$ of about 260). Follows the contributions of the 636 and 1433 ones. Finally the 702, 802 and 1400 streams are found to have a small contribution $(Z H R \simeq 10-20)$. It is important to consider many particles in the stream: a better sampling of the stream assures more accurate results (see also Brown \& Arlt 2000).

Having done a fast computation with very small particle (radius between 0.05 and $0.1 \mathrm{~mm}$ ), we did not find any intersecton with the Earth orbit in 1998, unlike Ma et al. (2001) have suggested from radar observations. Thus this is still an open question.

The 1998 shower could have been far more active if the Earth had entered the center of a young stream, as in 1833.

\subsection{Leonids beyond 2002}

Table 5 gives Leonid forecasts from 2003 to 2100 . The streams taken into account here are those in the period 1333 to 1998 , and a few older ones (602 to 802). These last ones were added as test of the contribution of old streams.

We note in this table the abscence of a shower in 2007, from the 1932 stream, unlike Lyytinen \& Van Flandern (2000). In our simulations the whole stream will have already passed perihelion. This will be a good occasion to compare the different models.

Several showers appear here that have never been noticed before. One of these shower occurs in, 2009, i.e. eleven years after the comet return. The same charateristic is observed in 2043. The last major Leonid shower for the coming years is 2011. After that, only the annual component will be observable until the comet's return.

The most promising year will be 2034, with 4 encounters with relatively young streams giving rise to storms.

The 2069 shower is particular because the encountered stream (636) is highly perturbed by planets. It extends in the direction perpendicular to the cometary orbit. The 2069 shower is expected to last 24 hours, but the $Z H R$ value for such a perturbed stream is very low.

2066 and 2098 will produce fewer showers.

\section{Discussion}

\subsection{Strength of the model}

The strength of the model is that no assumption of the $Z H R$ profile or radial density of the stream was made. All the assumptions of the cometary model are constrained by observations of the coma, via the $[A f \rho]$ parameter.

The evolution of the meteoroid streams does not assume any specific acceleration at specific points of the orbit, like perihelion. The ejection velocity is taken from one of the latest and most complete hydrodynamical models (Crifo \& Rodionov 1997).

The determination of the encountered profile of the shower is possible, but requires further investigation. The number of encountered particles must be sufficient to compute a density at several points of the trajectory of the Earth.

The time of the showers is well constrained and in accordance with observations. The level of the showers is more uncertain, but still remains within 20 percent of the observed value range.

This method allows us to estimate the size distribution of cometary dust, based on meteor shower observation. Usually this distribution is computed on the base of comae observations.

\subsection{Limitations of the model}

Figure 1 shows that if a stream is far outside the Earth's orbit, the second space criterion will cause an undersampling of the cloud. The calculation of the spatial density becomes then unreliable.

Forecasts are sensitive to the size distribution index $s$, considered constant over the whole size range. In reality this may be more complicated.

We also have assumed that the comet outgasing process is always the same. We know that a comet can change from a return to another. 55P/Tempel-Tuttle is a faint comet, that has not been well observed. Further observations during the next return of the comet, in about 30 years may help clarify this issue.

Figure 1 representing descending nodes of selected particles shows that the stream extends in a direction perpendicular to the cometary orbital plane. Observations show that the peaks are not that wide. On the other hand the 2001 shower had a very high continuous component. This suggests a very complicated structure of the streams. Some investigations in this field were done by Jenniskens (2002a) and are still ongoing.

Finally, the cometary features can vary from one return to another, like comet $2 \mathrm{P} /$ Encke for example. Such a refinement is not yet included in the model, but can easily be added. 
Table 5. Leonid forecasts from 2006 to 2100 .

\begin{tabular}{ccccc}
\hline \hline Year & Stream & D/M & h:mn & ZHR \\
\hline 2006 & 1932 & $19 / 11$ & $06: 33$ & 600 \\
2007 & 1733 & $20 / 11$ & $01: 15$ & 10 \\
2008 & 1466 & $17 / 11$ & $01: 32$ & 450 \\
\hline 2009 & 1466 & $17 / 11$ & $21: 44$ & 500 \\
2009 & 1533 & $17 / 11$ & $22: 02$ & 250 \\
2009 & 1567 & $17 / 11$ & $07: 29$ & 500 \\
\hline 2011 & 1800 & $16 / 11$ & $22: 04$ & 500 \\
2012 & 1400 & $20 / 11$ & $08: 57$ & 20 \\
2014 & 1567 & $21 / 11$ & $08: 25$ & 60 \\
2015 & 636 & $23 / 11$ & $02: 51$ & 100 \\
2015 & 769 & $19 / 11$ & $22: 52$ & 20 \\
2019 & 736 & $09 / 11$ & $17: 44$ & 40 \\
\hline 2028 & 1300 & $19 / 11$ & $13: 35$ & 40 \\
2033 & 1899 & $17 / 11$ & $22: 05$ & 800 \\
\hline 2034 & 1433 & $18 / 11$ & $13: 13$ & 45 \\
2034 & 1633 & $19 / 11$ & $03: 37$ & 350 \\
2034 & 1666 & $19 / 11$ & $04: 39$ & 1350 \\
2034 & 1699 & $19 / 11$ & $05: 46$ & 1500 \\
2034 & 1767 & $18 / 11$ & $22: 43$ & 1050 \\
2034 & 1800 & $19 / 11$ & $00: 43$ & 70 \\
2034 & 1833 & $19 / 11$ & $02: 37$ & 90 \\
2034 & 1866 & $19 / 11$ & $05: 45$ & 20 \\
2034 & 1932 & $18 / 11$ & $03: 34$ & 2000 \\
\hline 2035 & 1366 & $19 / 11$ & $18: 01$ & 110 \\
2035 & 1633 & $20 / 11$ & $06: 11$ & 920 \\
\hline & & & &
\end{tabular}

\begin{tabular}{ccccc}
\hline \hline 2035 & 1666 & $20 / 11$ & $07: 30$ & 15 \\
2035 & 636 & $19 / 11$ & $18: 21$ & 90 \\
\hline 2036 & 1433 & $18 / 11$ & $21: 55$ & 10 \\
2036 & 1466 & $18 / 11$ & $21: 58$ & 260 \\
2036 & 1633 & $19 / 11$ & $16: 48$ & 20 \\
2036 & 1666 & $19 / 11$ & $19: 58$ & 20 \\
\hline 2037 & 1366 & $19 / 11$ & $12: 57$ & 140 \\
2037 & 1800 & $19 / 11$ & $19: 59$ & 460 \\
\hline 2038 & 1800 & $20 / 11$ & $15: 15$ & 80 \\
2038 & 1833 & $20 / 11$ & $22: 19$ & 50 \\
2039 & 1767 & $21 / 11$ & $03: 07$ & 120 \\
\hline 2042 & 1400 & $18 / 11$ & $13: 04$ & 180 \\
2043 & 1433 & $18 / 11$ & $17: 15$ & 50 \\
2043 & 1433 & $19 / 11$ & $19: 25$ & 180 \\
2043 & 1400 & $19 / 11$ & $17: 30$ & 600 \\
2044 & 1567 & $18 / 11$ & $02: 46$ & 100 \\
2047 & 1567 & $21 / 11$ & $18: 41$ & 60 \\
\hline 2069 & 636 & $22 / 11$ & $04: 07$ & 20 \\
2069 & 1433 & $19 / 11$ & $08: 43$ & 200 \\
2077 & 1333 & $21 / 11$ & $09: 27$ & 80 \\
2077 & 1400 & $20 / 11$ & $04: 24$ & 450 \\
2079 & 736 & $19 / 11$ & $17: 09$ & 150 \\
2081 & 669 & $19 / 11$ & $22: 47$ & 60 \\
\hline 2093 & 1600 & $20 / 11$ & $15: 20$ & 70 \\
2095 & 769 & $21 / 11$ & $04: 44$ & 30 \\
2095 & 769 & $20 / 11$ & $08: 52$ & 20 \\
\hline & & & & \\
\hline
\end{tabular}

\section{Conclusion}

Meteor shower predictions give satisfactory results. Time of maxima based on the median position of the nodes give an accuracy of a few minutes to a few tens of minutes in less favorable cases. These results are in good agreemeent with those done by McNaught \& Asher (1999) and Lyytinen et al. (2001). In the case of very old streams that extend widely, a selection of particles is necessary. However, our results suggest a wider shower than observed.

Predictions of the level of the shower based on the photometry of the parent body give satisfactory results. We were able to reproduce the observations of past years. Historical observations give contradictory results, but the real ZHRs are hard to define. In particular we note the good agreement between our results and Jenniskens (2002a)'s work about the 1833 storm. The ratio between reported observations and predictions for this year is about 5. Generally, results give far lower values for historical showers than reported or previously computed.

By fitting the size distributon index $s$, we were able to provide Leonid shower ephemerides until 2100. Next storms are expected in 2034 and 2035.

The advantage of this approach is that it allows us to only fit one parameter, $s$. The value found here is consistant with observations of comets. However this fit is global and does not take into account the variation of activity of the comet. These are difficult to estimate unless the comet is often observed, which is not the case of $55 \mathrm{P} /$ Tempel-Tuttle.

Acknowledgements. This work could not have been done without support of CINES for the simulations. We would like to thank the whole

team. We also thank the other "meteor predictors": D. Asher, R. McNaught, E. Lyytinen, T. van Flandern and P. Jenniskens. This work is supported by CNES (French Space Agency).

\section{References}

Arlt, R., \& Brown, P. 1999, WGN, J. Int. Meteor Org., 27, 267

Arlt, R., \& Gyssens, M. 2000, WGN, J. Int. Meteor Org., 28, 195

Arlt, R., Bellot Rubio, L., Brown, P., \& Gyssens, M. 1999, WGN, J. Int. Meteor Org., 27, 286

Arlt, R., Kac, J., Krumov, V., Buchmann, A., \& Verbert, J. 2001, WGN, J. Int. Meteor Org., 29, 187

Arlt, R., Krumov, V., Buchmann, A., Kac, J., \& Verbert, J. 2002, WGN, J. Int. Meteor Org., 30, 205

Asher, D. J. 2000, in Proc. Int. Meteor Conf., 5

Asher, D. J., Bailey, M. E., \& Emel'Yanenko, V. V. 1999, MNRAS, 304, L53

Beech, M., Hughes, D. W., \& Murray, I. 2001, Earth Moon and Planets, 84, 143

Brown, P. 1999, Icarus, 138, 287

Brown, P., \& Arlt, R. 1998, WGN, J. Int. Meteor Org., 26, 11

Brown, P., \& Arlt, R. 2000, MNRAS, 319, 419

Campbell-Brown, M. D., \& Koschny, D. 2004, A\&A, 418, 751

Crifo, J. F., \& Rodionov, A. V. 1997, Icarus, 127, 319

Everhart, E. 1985, in Dynamics of comets: Their origin and evolution, ed. Carusi \& Valsecchi, 185

Fulle, M., Levasseur-Regourd, A. C., McBride, N., \& Hadamcik, E. 2000, AJ, 119, 1968

Göckel, C., \& Jehn, R. 2000, MNRAS, 317, L1

Gural, P. S., \& Jenniskens, P. 2000, Earth Moon and Planets, 82, 221 
Hainaut, O. R., Meech, K. J., Boehnhardt, H., \& West, R. M. 1998, A\&A, 333, 746

Hughes, D. W. 1995, Earth Moon and Planets, 68, 31

Jenninskens, P. 2001, WGN, J. Int. Meteor Org., 29, 165

Jenniskens, P. 2002a, in Proc. of Asteroids, Comets, Meteors - ACM 2002. Int. Conf., 29 July-2 August 2002, Berlin, Germany, ed. B. Warmbein (Noordwijk, Netherlands: ESA Publications Division) ESA SP-500, 117

Jenniskens, P. 2002b, WGN, J. Int. Meteor Org., 30, 218

Jorda, L., Crovisier, J., \& Green, D. W. E. 1992, Bull. Amer. Astron. Soc., 24, 1006

Kresak, L. 1993, A\&A, 279, 646

Lamy, P., Znojil, V., \& Biver, N. 1998, IAU Circ., 6851, 2

Lyytinen, E., Nissinen, M., \& van Flandern, T. 2001, WGN, J. Int. Meteor Org., 29, 110

Lyytinen, E. J. 1999, Meta Res. Bull., 8, 33

Lyytinen, E. J., \& Van Flandern, T. 2000, Earth Moon and Planets, 82, 149

Ma, Y., He, Y., \& Williams, I. P. 2001, MNRAS, 325, 457

Marsden, B. G. 1969, AJ, 74, 720
McNaught, R. H., \& Asher, D. J. 1999, WGN, J. Int. Meteor Org., 27, 85

Murray, I. S., Hawkes, R. L., \& Jenniskens, P. 1999, Meteor. Planet. Sci., 34, 949

Sykes, M. V., Lebofsky, L. A., Hunten, D. M., \& Low, F. 1986, Science, 232, 1115

Uchiyama, S. 2002, WGN, J. Int. Meteor Org., 30, 47

Vaubaillon, J. 2002, WGN, J. Int. Meteor Org., 30, 144

Vaubaillon, J. 2003, Ph.D. Thesis, Observatoire de Paris

Vaubaillon, J., \& Colas, F. 2002, Proc. of Asteroids, Comets, Meteors - ACM 2002. Int. Conf., 29 July-2 August 2002, Berlin, Germany ed. B. Warmbein (Noordwijk, Netherlands: ESA Publications Division), ESA SP-500, 181

Vaubaillon, J., \& Colas, F. 2005, A\&A, 431, 1139

Vaubaillon, J., Lyytinen, E., Nissinen, M., \& Asher, D. 2003, WGN, J. Int. Meteor Org., 31, 131

Vaubaillon, J., Lyytinen, E., Nissinen, M., \& Asher, D. J. 2004, WGN, J. Int. Meteor Org., 32, 125

Williams, I. P. 1997, MNRAS, 292, L37

Wu, Z., \& Williams, I. P. 1993, MNRAS, 264, 980 